But these affections may possibly be only functionally nervous; they are not always connected with organic changes in the nervous centres. Contracture is sometimes congenital, but more commonly consecutive to nervous diseases, either with organic alteration, or purely nervous. This latter kind, which I have ascertained, as well as French physicians, by dissection, is termed by the latter (Rilliet and Barthez,) the essential contracture, which I have seen as the consequence of convulsions, purely nervous. Most commonly we see this state of permanent contracture of the flexors, connected with only a degree of paralytic weakness of the affected extremity or extremities. Complete paralysis, on the contrary, of the flexors as well as extensors, is almost always the result of transudation or softening If this takes place in one hemisphere of the brain, then the paralysis extends over the whole length of the opposite half of the body, from the muscles of the face to those of the foot.

The anus, soon after birth, offers an abnormity which we call atresia ani, or imperforate anus, sometimes without the least opening, at other times there is a small opening, leading to the rectum. I have seen no more than eight similar cases in the whole extent of my practice. The prolapsus ani, a frequent consequence of dysentery in children, shows us a part of the mucous membrane of the rectum. As there is always a degree of strangulation caused by the sphincter, a considerable passive congestion is necessarily present in the prolapsed mucous membrane; but sometimes we perceive a swollen surface, with much granulous appearance, the follicles swollen or ulcerated, which are all symptoms of inflammation. On the contrary, redness at the orificium ani, with erosions or ulceration, is most commonly the consequence of the irritating quality of discharge, or of uncleanness, and is seldom dependent on pure intestinal inflammation. I have been satisfied that the latter circumstances gives sometimes rise even to luxuriations like flat tubercles, independent of syphilis; and these luxuriations at the orificium are frequently surrounded by eczematous eruptions and ulcerations, extending over the contiguous parts of the thighs; this is in particular the case in scrofulous children abandoned to uncleanness. I have succeeded in curing hundreds of similar cases without antiphlogistic means.

The skin.-You need not to be referred in this place to the various chronic and acute eruptions of the skin; these, and ulcerations, or any other local affections of the derma, are easily recognizable, and have their more or less clear signification, local and constitutional. The red foetal colouration of the skin sometimes persists for some weeks after birth; and I remember a case where a practitioner took it for erysipelas. Cyanosis, if the consequence of the abnormal persistence of the foetal blood-communications, gets its full signification by auscultating, in which case a bellows murmur will never fail. But $I$ bave seen cases of cyanosis in children dependent upon gastric disorder, or rather derangement in the portal system. Yellow colouration, if primary, denotes bilious derangement, but frequently after erysipelas or erythema follows a degree of icterus during the course of recovery, which yellowness is without signification. Paleness of the skin, with proportional want of redness of the mucous membrane of the eyelids, the tongue, and lips, signifies chlorosis, which I met with in some cases even about the second year of age, and ascertained it by auscultation and the presence of the carotid souffle.

The temperature of the skin, dryness, or perspiring state, heat or coolness of it, are of the same signification as in adults, only that there is more variability of temperature in children. The high degree of dry and burning skin, proper to typhoid or putrid fevers, I never met with in the first years of childhood.

Next we shall speak of the pulse and then of some other symptoms.

\section{CA-SES IN MIDWIFERY,} WITH REMARKS.*

By THOMAS RADFORD, M.D., F.R.C.P., EDIN., \&c., \&c., \&c.

Consulting Physician to the Manchester and Salford Lying-in Hospital.

The following case was most kindly and liberally given to me by James Braid, Esq., M.R.C.S., Edin., C.M.W.S., \&c. \&c., for the purpose of publication:-

\section{Cas e VI.}

\section{Cesarean Section.-(Unsuccessful.)}

“ About one o'clock, P.M., on the 15th of June, 1847, I was requested to go to Wilmslow, in Cheshire, to perform the Cæsarean section in the case of a poor woman named Mrs. Toft, who had been in labour since early on the morning of the 12th, that was three days and a half, and for whom the surgeons in attendance considered there was no hope of relief otherwise than by such operation. I consequently started by the first railway train, accompanied by my son, and arrived at the house of the patient about half-past two o'clock. The patient was said to be about 30 years of age, and had been married twenty-one months. She became pregnant shortly after marriage, but aborted at the third month. On the present occasion she had arrived at the full term of utero-gestation before calling for professional aid, which indeed she did only after labour had commenced, which was early on the morning of the 12th. Mr. Mayson, surgeon, of Wilmslow, had attended her alone from Saturday morning till Monday morning, when he had Mr. Dean, surgeon, of the same place, associated with him, who continued his attendance, along with Mr. Mayson, up to the period when Mr. Dean came to request my aid. The patient had always been of a feeble constitution, with fair complexion, but now she was excessively pale and exhausted, and was much disfigured by a large bronchocele. Her 
pulse was very rapid and feeble. On examination, the first object which attracted attention was the arm of a well-developed child protruding from the vagina, proving it to be a case of shoulder presentation. The bones of the outlet of the pelvis were so crushed together that there was scarcely room for one finger to pass by the side of the protruding arm, so as to make an examination. The arm being pushed up, I ascertained that the rami of the pubes were so closely approximated that a finger placed edgeways could not reach the symphysis pubis; and the tubera ischii were only about an inch apart, for there was no point where two fingers placed side by side could pass when directed transversely. Indeed, owing to the close approximation of the rami pubis, tubera ischii, and os coccygis, there was barely room sufficient to permit two fingers to pass the outlet of the pelvis in the antero-posterior direction. Owing to the shallowness of the pelvis, however, which must hare been originally of small dimensions, I was the more readily enabled to reach and determine the dimensions of the brim. I ascertained that there was not as much available space in the antero-posterior direction as to permit the points of two fingers, laid side by side, to pass the brim of the pelvis, excepting about half an inch exactly opposite the symphysis pubis, and there the fingers had barely room to pass. Beyond this, on either side, there appeared to be little more than an inch of available space in the antero-posterior direction. The transverse diameter might exceed three inches, but then it was crescentic in form, which, of course, made it completely unavailable for delivery. My son having also made an examination of the patient, and made a similar estimate of the relative position of the bones of the pelvis at the brim and outlet, which were, moreover, firm and unyielding, we had no difficulty, in consultation with the other two surgeons, Mr. Mayson and Mr. Dean, in arriving at the conclusion that the woman must die, undelivered, if we did not instantly resort to the Cæsarean section. With such deformity as existed here in the pelvic bones it must have been all but impossible to have broken down and extracted a fullydeveloped child, (which from the protruding arm this evidently was), even in a vigorous patient; but in a feeble woman like the one in question, exhausted to the last degree by the length of time she had been in labour, and the violence and acute suffering which she experienced from the pains, even up to the period when we were with her, it would have been perfectly futile and absurd to have made any attempt of the sort. The extreme violence and excruciating agony which the patient was suffering from the pains rendered it the more desirable that the operation should not be unnecessarily delayed; and we, therefore, stated our views of the whole bearings of the case fully and fairly to the friends, and obtained their consent, and subsequently the patient's also, when I proceeded to perform the operation in the usual manuer, at three o'clock. I deem it quite unnecessary to occupy your time by giving any details of the operation, for, although a formidable and a most important one, and one which ought only to be performed from the necessity of the case, still, quasi a surgical operation, it involves comparatively little difficulty to those who are well acquainted with the anatomy of the parts, and are in the frequent habit of operating. A very few minutes sufficed to make the necessary incisions and to extract the child, the placenta and the coagula were found in the uterus. All this and the stitching, dressing, and replacing the patient in bed, certainly did net exceed ten minutes; and the whole pain sustained by the patient in consequence of the operation, did not appear much to exceed a single pain, such as she had in our presence, from the throes of nature, before we proposed the operation.

"The infant was large, and well developed, but was dead, obviously from previous detachment of the placenta, for it was found quite detached, and surrounded by coagula, which at once accounted for the exsanguine appearance of the mother, as well as for the death of the child. There had been very little coloured discharge per vaginam, the egress having been completely closed by the shoulder of the child being impacted into the brim of the pelvis. Very little blood was lost by the incisions made during the operation, and very little passed per vaginam, subsequently. After the operation the patient seemed to suffer no more pain, but she passed quietly away, from exhaustion, five hours and a half after the operation.

" On the 17th I went over, accompanied by my son and another medical friend, for the purpose of having a post-mortem. Mr. Dean was also with us. We had almost been too late, as the company had assembled before our arrival for the purpose of interring the body ; they consented to postpone it a very short time, to allow us to make an inspection, but we were necessarily compelled to be very circumspect, as we were closely watched, and were thus prevented the opportunity of possessing ourselves of the pelvis; however, I had used the precaution of taking some plaster of Paris with us, and thus we were enabled to take an accurate model of the inlet of the pelvis; from this it was satisfactory to find that the estimate made of the brim of the pelvis previous to undertaking the operation had been very correct, as the following measurements of the cast prove :-The diameter of the brim of the pelvis, from the symphysis pubis to the lumbar vertebra, was one inch and three-eighths, and beyond that on the left side it abruptly diminished to nine-eighths of an inch and from that to half an inch, and on the right side from an inch and a quarter to an inch. The transverse diameter from right to left was three inches and a half, but of this there were not more than two inches, which would admit a ball to pass which exceeded an inch in diameter. But, as already stated, the brim had assumed a crescentic form, so that when two straight parallel lines were drawn across the pelvis, they only showed two inches by one inch as the largest available space. Under these circumstances, therefore, it is quite obvious that delivery, by mutilation of the infant, conld not have been undertaken with an hope of success, at any stage of the labour, however vigorous the patient might have been; how much less so, then, with such a con- 
stitution as we had here to encounter, and with a shoulder presentation, too? The cavity of the pelvis was so small as not to admit a body greater than a lemon. At the left side and anterior part of the fundus uteri the walls of the uterus were fully an inch and a half in thickness, whilst a considerable portion on the posterior and right side was attenuated in an extreme degree, so that from this circumstance, and the violent and cutting character of the pains witnessed by us, had she been left a short time longer untouched, in all probability rupture of the uterus, and death, would have been the result. Very little blood hal escaped per vaginam subsequently to the operation, and there was only a small clot found within the cavity of the uterus. Here, then, is a case which, I think, fully justifies the Cæsarean section, for no medical man bad been consulted until the woman was in labour at the full term of utero-gestation, and with physical attributes which rendered it impossible for her to be delivered in any other manner than by the Cæsarean section. The only cause of regret is, that this had not been undertaken immediately after labour commenced, for in that case there is every reason to believe that the life of the infant would have been spared, with a tolerable chance of safety for the mother also. This alternative had been proposed to the patient before I was sent for, but she obstinately held out against submitting to any such operation. I consider it but an act of justice to Mr. Dean and Mr. Mayson to record this fact, and that it took some management on my own part to obtain her consent at last. Although the operation failed to save the life of mother or child, still it relieved her of suffering for the last five hours and a half of her life, and her friends the pain of hearing her piercing screams, and witnessing the agonizing throes which accompanied the unavailing efforts of nature to relieve her from her perilous condition."

\section{Dr. Radford's Remarks.}

Malacosteon doubtless was the disease which caused the distortion of the pelvis of this poor creature. There are no data whereby to judge when its ravages on the bones were commenced. Some of the contingent circumstances which happened, clearly prove the truth of those statements I made a short time ago (vide Provincial Medical and Surgical Journal, No. 7, April, 185], page 175. It was her first labour; and we have no evidence to show that any symptoms existed either before or during her pregnancy, to induce her to place herself under medical treatment. The obstetrician was completely ignorant of the physical and organic condition of the pelvis until after labour had commenced. It is, then, indisputably true, that no other operation but the Cæsarean could possibly or safely be performed for her delivery. The great mischief of procrastinating the operation is emphatically proved by the result. Several serious evils arising from the protracted labour are noticed by $\mathrm{Mr}$. Braid. The death of the infant was undoubtedly produced by it, and most likely that of the mother. The internal flooding, the complete separation of the placenta, and the attenuated state of one portion of the structure of the uterus-which no doubt would have ended in its rupture-are solely to be attributed to delay.

In Cases I. and V. I have mentioned that violent irregular uterine contraction happened during the extraction of the infant, which I stated depended on the partial or complete detachment of the placenta. In the foregoing case, however, nothing of the kind took place, although this organ was lying loose in the uterus. Did the internal bleeding (which was so great as to bleach the general surface) act on the uterine tissue, and so influence its contractility? Was the absence of this spasm (when its supposed cause was present) owing to the extreme degree of attenuation of the uterine tissue?

\section{CASES IN MIDWIFERY.}

By J. KENWORTHY, M.R.C.S., MANchester.

Read at the Nineteenth Anniversary Meeting, held at Brighton, August 13,1351.

THE following extracts from my midwifery notes being cases of uterine hæmorrhage, in connection with placenta prævia, may possibly be interesting to some of the members of the Association; some of them occurred many years since, and the minutice are not all recorded, for eighteen years of the time in which the earliest cases happened I was a good deal occupied in the hospital wards of the workhouse, but did not meet with any case of importance of this description in that class of practice.

Case 1.-March 7th, 1819.-Mrs. B. One edge of the placenta presented. Pressure of the fotal head lessened the hæmorrhage, and the labour terminated in the natural way. She was faint after it was over, and seventy drops of laudanum, with a little brandy, were administered, in tea. The mother and child both did well.

Case 2.-January 30th, 1821.-Mrs. W. Was seven months advanced; there was hæmorrhage a week previously, which subsided under the use of acids; quiet, horizontal position, and cold externally applied. At ten P.M. on the 29th hæmorrhage returned. I found the placenta presenting, but the cervix uteri would not admit my finger through it, being thick and unyielding. The same means as before, with the addition of ten drops of tincture of opium every hour There was no uterine action. I enjoined perfect quiet, a free admission of air, and to drink cold lemonade. At five o'clock on the morning of the 30th no natural change; the hæmorrhage was less. At 10 A.M. the bleeding was much increased, and she was faint. The cervix was more yielding, and partly dilated. I thought it wrong to delay, and introduced my hand very quietly round the placenta, broke the membranes, and delivered by the feet. There was very little uterine action throughout. She was much 